\title{
Development of an Evidence and Gap Map (EGM) of interventions to increase smoking cessation: A study protocol
}

\author{
Arifuzzaman Khan ${ }^{1,2}$, Catherine King ${ }^{3}$, KM Saif-Ur-Rahman ${ }^{4,5}$, Gulam Khandaker ${ }^{1,2}$, Sheleigh Lawler ${ }^{1}$, Coral Gartner ${ }^{1}$
}

\begin{abstract}
Smoking remains one of the leading risk factors contributing to the global burden of disease. Sub-optimal implementation of evidence-based tobacco control and smoking cessation practice is a major challenge despite a substantial evidence base for interventions to increase smoking cessation globally. We aim to develop an Evidence and Gap Map (EGM) to collate the existing evidence and identify the gap in research on interventions to increase smoking cessation worldwide. A conceptual framework was developed followed by the formulation of a search strategy with key search terms and search period (1970 - date of search). The search will be conducted in relevant bibliographic databases (e.g. MEDLINE, Embase, SCOPUS), systematic reviews databases (e.g. Cochrane Library, Joanna Briggs systematic reviews, EPPI-Reviewer) and impact evaluation databases (e.g. 3ie Impact Evaluation repository and Cochrane tobacco addiction group specialized register) with support from a research librarian. Subsequently, two coders will screen and retrieve systematic reviews and individual impact evaluation studies. The adapted SURE (Supporting the Use of Research Evidence) checklist will be used to evaluate the quality of the included systematic reviews. A narrative synthesis from the systematic review findings and line listing of the impact evaluations will form the basis of this EGM. The EGM report will be presented in an interactive visual format. The proposed EGM will organise the pieces of evidence generated in systematic reviews and impact evaluations on smoking cessation interventions and identify the current research gaps, if any. The findings will inform evidence-based practice and future research.
\end{abstract}

\section{AFFILIATION \\ 1 School of Public Health, The University of Queensland, Brisbane, Australia \\ 2 Central Queensland Public Health Unit, Central Queensland Hospital and Health Service, Rockhampton, Australia \\ 3 Faculty of Medicine and Health, The Children's Hospital at Westmead Clinical School, The University of Sydney, Sydney, Australia \\ 4 Department of Public Health and Health Systems, University of Nagoya, Nagoya, Japan \\ 5 Health Systems and Population Studies Division, International Centre for Diarrhoeal Disease Research, Dhaka, Bangladesh}

\section{CORRESPONDENCE TO}

Arifuzzaman Khan. School of Public Health, The University of Queensland, Herston OLD 4006, Brisbane, Australia. E-mail: mdarifuzzaman.khan@uqconnect. edu.au ORCID ID: https://orcid. org/0000-0003-4770-8308

\section{KEYWORDS \\ tobacco smoking, smoking cessation, smoking cessation intervention, evidence and gap map (EGM), systematic review, impact evaluation}

Received: 18 March 2020

Revised: 16 June 2020

Accepted: 17 June 2020

\section{INTRODUCTION}

Tobacco smoking remains the second leading risk factor (top behavioural risk factor) contributing to the global burden of disease, which is estimated to include 7.1 million (95\% CI: 6.83-7.37 million) deaths and 182 million (95\% CI: 173-193 million) DALYs in
$2017^{1}$. The number of smokers worldwide is estimated to be 1.1 billion, which is expected to reach 1.6 billion by $2025^{2}$. Reducing the global toll from smoking will require preventing young people from taking up smoking and increasing the number of smokers who quit, with epidemiological modelling showing that a 
faster impact on smoking prevalence can be achieved by increasing smoking cessation rates ${ }^{3}$. Hence, increasing smoking cessation remains a public health priority. A strong evidence base supports a range of individual-level and population-based strategies to increase smoking cessation by encouraging smokers to make a quit attempt and helping them to be more successful in these attempts in the short- and longterm. Most high-income countries (HICs), such as Australia, Canada, New Zealand, USA, UK and European countries have adopted a comprehensive approach to tobacco control by implementing a range of strategies to encourage and support smokers to quit. However, much more could be done to increase smoking cessation, particularly in low- and middleincome countries (LMICs) since most of the smokers are located in LMICs ${ }^{4}$.

The World Health Organization's Framework Convention on Tobacco Control (WHO-FCTC) has facilitated the worldwide dissemination of evidencebased strategies that increase smoking cessation ${ }^{5}$. To assist countries to implement the FCTC, the WHO has produced guidance on a package of evidencebased strategies known as MPOWER (Monitor tobacco use and prevention policies; Protect people from tobacco smoke; Offer help to quit tobacco use; Warn about the dangers of tobacco; Enforce bans on tobacco advertising, promotion and sponsorship; Raise taxes on tobacco $)^{6}$. According to the WHO, the introduction of MPOWER has had a significant global impact ${ }^{5}$. Implementation of the interventions that are reflected by this framework has been found significantly associated with the reduction of global smoking prevalence ${ }^{7}$. The FCTC contains 17 Articles that describe a range of evidence-based tobacco control strategies ${ }^{5}$. WHO employed an expert group to evaluate the impact of FCTC over a decade (2005-2015) ${ }^{5}$. The group found that significant and rapid progress has occurred with the implementation of several FCTC interventions (i.e. comprehensive smoke-free laws, pictorial health warnings, mass media campaigns and bans of sales to minor groups) over the decade ${ }^{5}$. However, moderate and slow progress has been observed in implementing the interventions that help to increase smoking cessation ${ }^{5}$. Furthermore, progress in FCTC implementation was highly variable across countries and regions with greater progress in high-income countries, such as Australia, Canada, New Zealand, USA and UK, but poorer progress in $\mathrm{LMICs}^{5}$. Systematic reviews provide evidence in support of interventions that increase smoking cessation, but not all interventions have been adopted in all countries for many reasons such as cost, access and weak enforcement ${ }^{5}$. Due to scarce resources in many settings, policymakers need to prioritise which interventions would be most appropriate and feasible to implement in their context in the short-, medium-, and long-term.

Good quality evidence generated from a systematic review can help policymakers to adopt evidence-based strategies. However, review findings can differ due to the variability of methods and reliability of study findings included in the reviews. A brief search on 17 February 2020 in the Cochrane Library Database for Systematic Reviews using the title 'Interventions to increase Smoking Cessation' identified 71 systematic reviews and 1941 trials. A systematic review reported that there were 45 articles published from the impact evaluation studies on smoking cessation interventions (i.e. generally on offering help to quit smoking including pharmacotherapy, health education and on warning of the dangers of tobacco) from low- and middleincome countries (LMICs) ${ }^{8}$. Other reviews focused on a specific type of intervention individually, varying from mass media, mobile phone-based interventions to community pharmacy interventions, exercise or acupuncture and hypnotherapy ${ }^{9-15}$. Whether the intervention was delivered alone or in combination with other interventions, such as pharmacotherapy or behavioural support, also varied $^{16-18}$. The reviews are also varied by the populations included, ranging from indigenous peoples, young people to nursing care patients ${ }^{16-18}$.

Moreover, few systematic reviews evaluated the effect of changes in health systems, policies and strategies in a different context ${ }^{19-21}$. Hence, individual systematic reviews have limitations that can inhibit effective translation into policy and practice. Furthermore, findings can differ between systematic reviews due to variability in methods and the selection of studies included in the review. For example, four reviews of the effect of incentives on smoking cessation outcomes came to different conclusions $^{20,22-24}$. 
The Evidence and Gap Map (EGM) is a new but increasingly popular approach for collating the evidence from systematic reviews and individual impact evaluation studies to inform researchers and policymakers ${ }^{25}$. The use of EGM facilitates evidence-based decision-making since it produces a better summary of the evidence from the available systematic reviews and removes the redundant and conflicting results of these reviews ${ }^{25}$. It contains a state-of-the-art pictorial tool to map the known effect and impact of different interventions and identify any research gaps that exist for those interventions for a specific theme ${ }^{25}$. EGM systematically selects articles and narrates the amplitude and limitations of each review or impact evaluation study ${ }^{25}$. Thus, it helps the audience, including non-specialists, to obtain a visual overview of the relevant evidence on the topic and to understand what evidence is missing. Therefore, EGM is emerging as a chosen tool for researchers in many fields because of its usefulness in prioritising research and translating it into practice and policy ${ }^{25,26}$.

To date and to the best of our knowledge, no EGM has been conducted to collate the evidence on the impact of smoking cessation interventions that offer assistance to quit smoking targeting high-, low-, and middle-income countries. In this context, we aim to develop an EGM on smoking cessation interventions.

\section{Aim and objectives}

Overall aim

To collate and review the evidence on interventions that increase smoking cessation and identify research gaps, if any, through an EGM process.

\section{Specific objectives}

1. To organise and outline the existing evidence on the effectiveness of interventions to increase smoking cessation.

2. To collate the evidence of the impact of smoking cessation interventions on smoking-related diseases, hospital admissions and deaths burden.

3. To summarise the existing evidence on the costeffectiveness of interventions to increase smoking cessation.

4. To describe the feasibility and acceptance of different interventions to increase smoking cessation.

\section{METHODS}

The EGM method maps out the existing systematic reviews and impact evaluations in a specific area of interest. It creates a visual framework that collates the available evidence on the interventions and outcomes of the topic. We have developed this EGM protocol following the International Initiative for Impact Evaluation (3ie) EGM guidelines and recommendations ${ }^{27}$. We will report the EGM findings according to the instructions of the Reporting standards for Systematic Evidence Syntheses (ROSES) ${ }^{28}$. We have developed the draft of a comprehensive search strategy based on the objectives and proposed conceptual framework. We will systematically search the literature following the developed search strategy (discussed later in a separate section) before conducting the screening process.

\section{Development of conceptual framework}

The 'inputs' of the interventions to increase smoking cessation are based on one of the six themes of the WHO MPOWER framework ${ }^{6}$, namely: Offer help to quit tobacco use. The conceptual framework (Figure 1) was developed based on the interventions that we considered for this EGM through literature reviews. The framework shows the possible impact (ranging from immediate to long-term outcomes) of the implementation of different smoking cessation interventions. This framework for creating, organising and structuring the EGM has been finalised through consultation among the research team members. The structure of the EGM framework is in the format suggested by the International Initiative for Impact Evaluation (3ie) EGM guidelines and recommendations ${ }^{27}$.

\section{Interventions}

A preliminary review of electronic databases (e.g. the Cochrane Library) and research team discussions identified at least 10 categories of interventions for this EGM. However, any category will be included in the EGM if it is found in the literature search. The interventions are itemised in a PopulationIntervention-Comparison-Outcome (PICO) structured table (Table 1).

\section{Outcomes}

We have divided the outcomes of smoking cessation 
Figure 1. Conceptual Framework for the EGM on interventions to increase smoking cessation
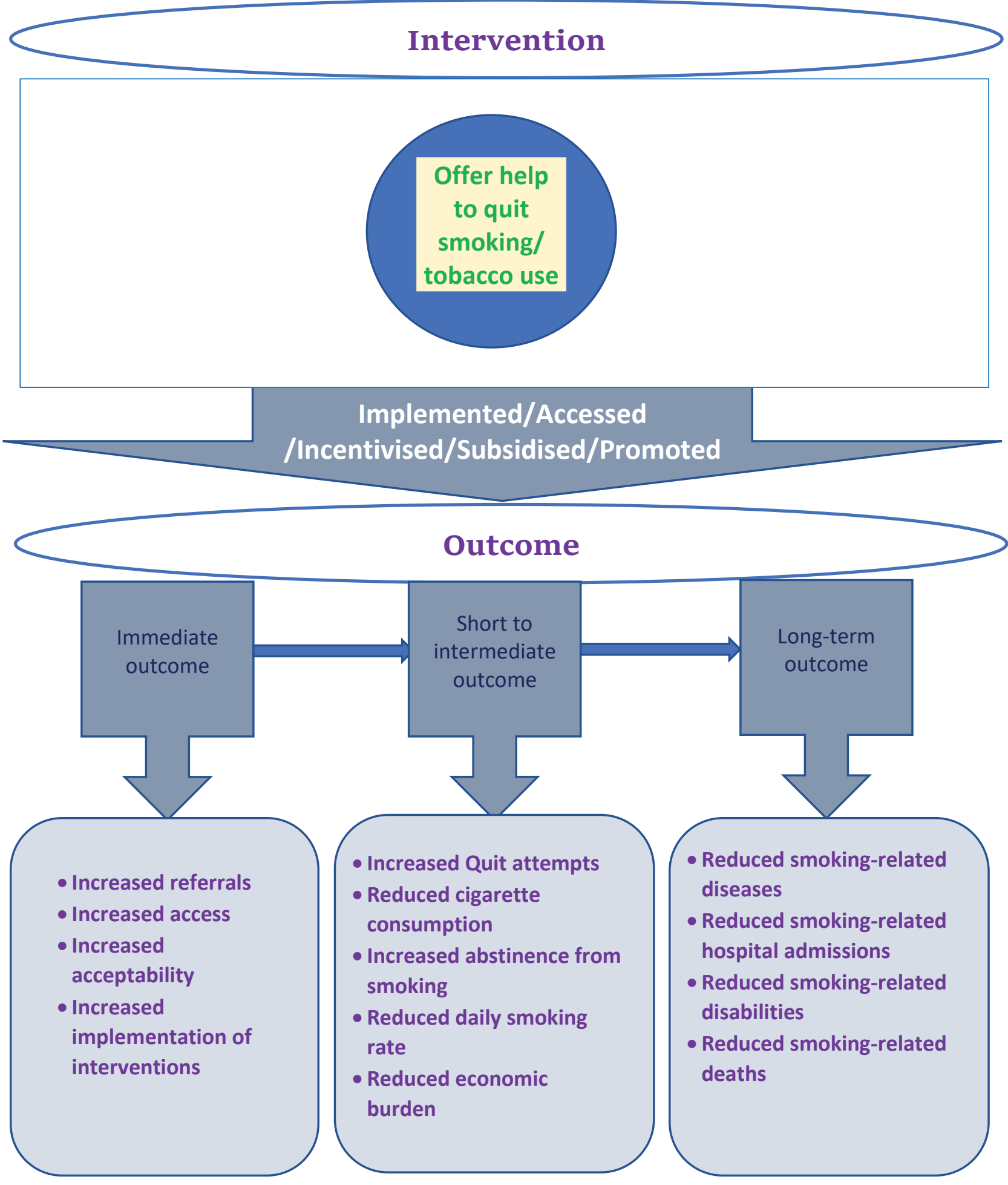
Table 1. Inclusion and exclusion criteria structured in PICO

\begin{tabular}{|c|c|c|}
\hline PICO & Inclusion criteria & Exclusion criteria \\
\hline Population & Those who currently smoke & Those who do not currently smoke \\
\hline Intervention & $\begin{array}{l}\text { A. Behavioural interventions } \\
\text { - Brief intervention } \\
\text { - Quit lines } \\
\text { - m-Tobacco cessation (e.g. text messaging, phone call) } \\
\text { - Intensive behavioural support } \\
\text { - Smoking cessation clinics } \\
\text { B. Pharmacological intervention } \\
\text { - Nicotine replacement therapies (NRTs) } \\
\text { - Non-nicotine pharmacotherapies (e.g. bupropion, varenicline) } \\
\text { C. Public health legislation } \\
\text { Policies as interventions to increase smoking cessation (e.g. smoke-free public place, } \\
\text { smoke-free hospitals) } \\
\text { D. Healthcare financing } \\
\text { Financing for promoting cessation activities (e.g. subsidised therapies, incentives, } \\
\text { insurance, training and education to healthcare personnel) } \\
\text { E. Specific interventions } \\
\text { Interventions specific to different phases of smoking cessation (i.e. motivation, } \\
\text { cessation, maintenance, relapse-recovery) } \\
\text { F. Interventions in different settings (e.g. primary healthcare centre, tertiary level } \\
\text { hospitals) } \\
\text { G. Alternative therapies } \\
\text { (e.g. acupuncture, yoga, exercise) } \\
\text { H. Campaign of promoting smoking cessation interventions }\end{array}$ & $\begin{array}{l}\text { Wider lifestyle interventions } \\
\text { not specific to tobacco smoking } \\
\text { behaviour }\end{array}$ \\
\hline Comparison & $\begin{array}{l}\text { Placebo } \\
\text { No intervention } \\
\text { Usual care } \\
\text { Minimal intervention } \\
\text { Pre-post comparison of impact } \\
\text { or outcomes }\end{array}$ & \\
\hline Outcome & $\begin{array}{l}\text { Primary outcomes } \\
\text { - Abstinence from smoking; self-reported or biochemically validated } \\
\text { - Consumption of cigarette; self-reported } \\
\text { - Intention to quit; the number of people setting a quit date } \\
\text { - Prevalence of smoking } \\
\text { Secondary outcomes } \\
\text { - Increased use of smoking cessation support as } \\
\text { immediate impact } \\
\text { - Smoking-related diseases } \\
\text { - Smoking-related hospital admissions } \\
\text { - Cost-effectiveness (e.g. costs, savings, incremental cost) } \\
\text { - Acceptability (i.e. proportion of smokers that continue to receive the treatment for } \\
\text { recommended duration) }\end{array}$ & \\
\hline
\end{tabular}

interventions primarily into three categories:

1) Immediate-term outcomes, which are reflected immediately after the exposure of intervention, and include positive changes in awareness for the benefit of smoking cessation, participation in smoking cessation education and training, number of referrals to support hub (e.g. Quitline), and use of smoking cessation treatments.
2) Short- to intermediate-term outcomes, which are reflected over 1-2 years after the interventions are implemented, comprise the changes in the number of quit attempts, daily smokers and exsmokers, and the rate of cigarette consumption and abstinence from smoking.

3) Long-term outcomes, which are reflected after 4-5 years from when the interventions are 
implemented, include smoking-related deaths, diseases, disabilities and overall health status of the population.

We will also consider the cross-cutting themes for this EGM, i.e. cost-effectiveness, the feasibility of implementation, and the acceptability by the smokers, of the smoking cessation interventions.

However, different outcomes are listed in a Population-Intervention-Comparison-Outcome (PICO) structured table (Table 1).

\section{Consultation meeting}

The investigator team comprises researchers with expertise in public health, tobacco control, psychology, public health physician, PhD student, familiar user of EGM method, research librarian and program people. In our investigator team meeting, we discussed the drafts of the conceptual framework, intervention/outcomes framework and PICO terms that are developed after a preliminary exploration of published policy and a few systematic reviews related to smoking cessation intervention. We have finalised the framework and search terms after discussion with all investigators and designated research librarian for this study. As this research involves published articles, it is exempted from ethical approval. We will disseminate the research findings including the interactive map at relevant conferences and via peerreviewed publications.

\section{Search strategy}

\section{Process}

Following the consultation with the research librarian (a co-author of this article) of the team, we have drafted the comprehensive search strategy for this EGM. During a scoping search phase, we identified the key search terms that are focused on interventions to increase smoking cessation with their different approaches and outcomes. The research librarian combined the key search terms into a search strategy for Ovid MEDLINE® (Supplementary Table S1). The scoping strategy was then reviewed by all team members and some additional terms added. We have checked the comprehensiveness of the search strategy through validating a few standard systematic reviews and impact evaluation articles prior to finalising the search terms. The final strategy will be used for other bibliographic databases, adapting the database- specific thesaurus and study-type terms, where necessary.

\section{Information sources}

We will implement the comprehensive search strategy to systematically search the relevant electronic bibliographic databases, systematic review databases, impact evaluation databases and relevant bilateral agencies (e.g. WHO, Centers for Disease Control and Prevention - CDC) websites. These will include: OVID MEDLINE, OVID Embase, CINAHL, Cochrane Library databases including Cochrane Tobacco Addiction Group (TAG) specialised register, PsycINFO and SCOPUS, Joanna Briggs Institute Systematic Reviews, Campbell Collaboration, EPPI reviewer and the 3ie database of systematic reviews and Impact Evaluation repository. We will search citations from the articles published in the websites of the bilateral national and international agencies (e.g. WHO, WHO FCTC and United States CDG).

\section{Selection criteria}

We will include both systematic reviews and impact evaluation studies for evaluating smoking cessation intervention within the Intervention/Outcome framework in our EGM to map the evidence and identify the gaps. Additionally, the impact evaluation studies, assessing the effectiveness and outcomes of tobacco control and smoking cessation interventions, will be included.

\section{General inclusion criteria}

- Any systematic reviews evaluating the effectiveness or outcomes of the interventions for tobacco control and smoking cessation;

- Impact evaluation studies including Randomised Controlled Trial (RCT), Quasi-experimental studies or pre- and post-trial that evaluated the effectiveness and or outcomes of tobacco control and smoking cessation interventions;

- Published literature in any language;

- Published between 1970 - date of search.

\section{General exclusion criteria}

- Any trial or review that does not measure the impact of a tobacco control or smoking cessation intervention;

- Any trial or review outlining the effect of smoking 
rather than the impact of tobacco control or smoking cessation interventions;

- Any ongoing trial and review that is not completed or not published yet;

- Non-systematic literature reviews;

- Letters to the editor, editorial comments etc.

Inclusion and exclusion criteria are also shown in a PICO structured table (Table 1).

\section{Article selection process}

We will employ the standard methods of two-stage screening while selecting the articles for inclusion (Figure 2). Initially, we will screen search results for relevance based on the title and abstract. Two reviewers will check the eligibility of each article independently. A third reviewer will review any documents or articles for which there is disagreement.

Figure 2. Two-stage article selection process

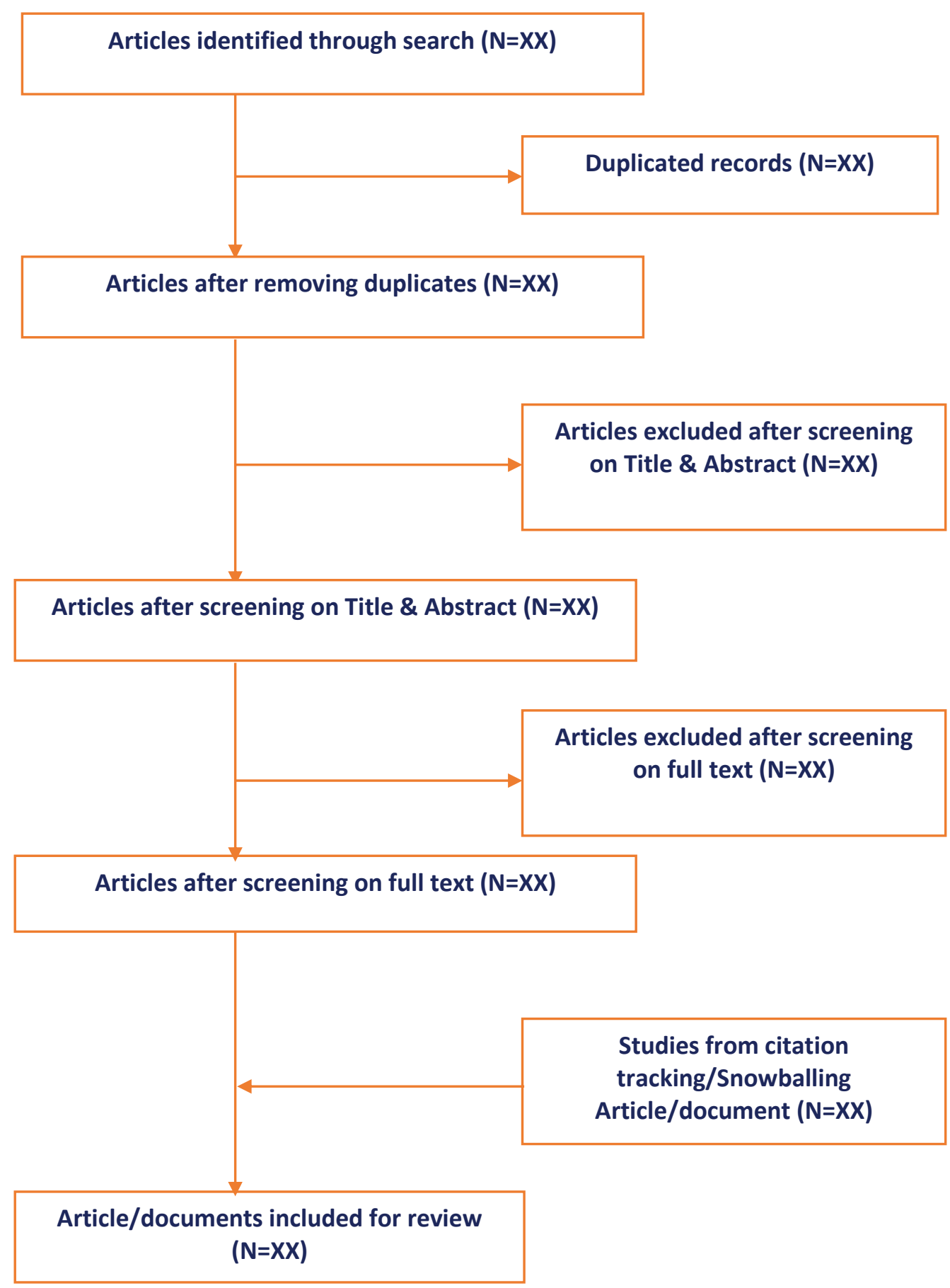


The final list of included articles will be determined by screening the full text. Screening for full text will also be conducted by two independent reviewers. In all stages, any disagreement between the reviewers will be discussed with a third senior reviewer until resolved. We will use pre-defined inclusion and exclusion criteria (described in this protocol; Selection Criteria section) in both stages of screening. A sequence of exclusion criteria will be applied to reduce the chance of subjectivity in prioritising exclusion criteria. We will report the exclusion of an article with proper documentation. Where there are multiple publications from a single study, all will be reported. We will use the ROSES flow diagram ${ }^{28}$ to present the summary of the process, including the numbers of excluded and included articles for this EGM.

\section{Data collection management}

We will organise the retrieved articles after the comprehensive literature search. We will create an Endnote library by incorporating all the available records found after the search of bibliographic databases. We will manage the references and information sources in EndNote. We will remove duplicate articles. We will develop a set of codes for the screening process to document the application of the inclusion and exclusion criteria.

\section{Data extraction management}

We will use a Microsoft Excel spreadsheet to store the extracted data from the included articles. The data extraction will indicate the data extraction date, time, name of the coder, publication year, geographical locations (e.g. country, city), target population, study settings, sample size, sociodemographic characteristics of the participants, study design or impact evaluation methods, description of the intervention, enrolment details, any attrition related information, any limitation reported in the article, and measurement of outcome including effect size with statistics mentioned in the article. We will collect the information relevant to assess the quality of the systematic reviews. Two coders will extract the data independently following the process of data extraction in the EGM. A third reviewer will review the process of data extraction randomly for about $5 \%$ of the extracted articles and resolve any disagreement between the independent reviewers.

\section{Quality assessment and management}

We have a comprehensive quality management plan for this EGM. We will start the coding for this EGM after a practice-run followed by a piloting session with the full EGM research team. During the piloting session, we will test screening and data extraction of one included systematic review and one included impact evaluation article in the team to ensure consistency in data extraction across the coders.

We will assess the quality of included systematic reviews using a standardised tool for critical appraisal used by the 3ie EGM group ${ }^{27}$. We will rank the systematic reviews with an overall rating of high-, medium-, or low-grade evidence regarding the confidence of their findings. Ranking will be performed by the coders using the checklist (Supplementary Table S2) adapted from SURE (Supporting the Use of Research Evidence) ${ }^{29}$. A third reviewer will resolve any dispute in quality assessment between the independent coders. The quality of the impact evaluation will not be assessed due to limitations in time and resources. We will, however, collect the information on the study design for each impact evaluation study.

\section{Data analysis}

We will produce a narrative synthesis by describing the interventions and outcomes of the systematic reviews and impact evaluations. We will generate a geographical information system (GIS) map showing the frequency distribution of the impact evaluation studies conducted in different countries in the world. We will tabulate the studies on smoking cessation interventions. We will classify the interventions into different categories according to the type of intervention (e.g. behavioural interventions, pharmacotherapies, policy and legislative interventions), phase of smoking cessation (i.e. motivation, cessation, maintenance, relapserecovery ${ }^{30}$ ), and setting (primary health care centre, tertiary level hospitals, community). We will list the different outcomes of the tabulated interventions. We will present the frequencies of studies conducted for various smoking cessation interventions along with their outcomes in a graph. Alongside, we will describe the key characteristics of the population for a particular intervention.

All efforts for the data analysis in the EGM will 
allow us to explore the existing evidence of smoking cessation interventions. It will provide us with the opportunity to identify the 'absolute evidence gaps' (i.e. no studies for a particular intervention) and 'synthesis gaps' (several impact evaluation literatures, but no high confidence systematic reviews). This gap will initiate a new systematic review or evidence synthesis study afterwards.

\section{EGM visualisation}

We will present the findings after the analysis of the extracted data in a visual-interactive format using 3ie's customised platform. We will attach a detailed report of the EGM with this platform. A computer programmer will help us to create this visual and dynamic platform from the data (e.g. intervention, outcome, study design for impact evaluation, systematic review confidence grade, geographical location) extracted for all included systematic reviews and impact evaluation studies. The colour grade of graph will indicate the evidence grade and the size of the plotting will indicate the number of studies to the audience.

\section{DISCUSSION}

EGMs synthesise the information that is available on what interventions are effective by specific themes. This method allows us to collect thematic evidence from systematic reviews and impact evaluations on the interventions and outcomes of the defined theme. EGM creates a visual presentation of the quantity and quality of systematic reviews and lists the impact evaluations.

\section{Potential research implications}

All efforts for the data analysis in the EGM will allow us to explore the existing evidence of smoking cessation interventions. It will provide us with the opportunity to identify the 'absolute evidence gaps' (i.e. no studies for a particular intervention) and 'synthesis gaps' (i.e. several impact evaluation literatures, but no high confidence systematic reviews). It creates demand for generating and synthesising new evidence. This gap analysis will also help prioritise research on those topics that require further empirical evidence. The EGM findings will assist policy makers to efficiently identify which smoking cessation interventions have the strongest evidence, and in which settings.

\section{Strengths and limitations}

Our EGM will employ a best practice model by preparing frameworks of intervention/outcome and PICO terms from the stakeholder consultation and by incorporating a systematic search strategy, specific inclusion and exclusion criteria, and systematically report all available studies. Our protocol is the first step to ensure compliance with best practice. This EGM will allow us to collate evidence published in any language and recognise any gap in current interventions for smoking cessation. The limitation of this research is that it will not synthesise any new evidence by doing a meta-analysis. However, it might identify one or more interventions to choose for new systematic reviews and meta-analysis. As the articles of impact evaluations are systematically searched and retrieved in the EGM, the next steps for new systematic reviews will be economical in terms of efforts, time and budget.

\section{CONCLUSION}

We will present the findings of the EGM in relevant meetings and symposia. We will publish the result in the form of web-based and interactive EGM in a website which will be open-access. We will publish the findings of the EGM in a separate manuscript. We will promote the EGM outputs and published manuscript via websites and social media platforms. Following the registration of this EGM into PROSPERO, the comprehensive search of all specified databases was completed in May 2020. The articles were imported into Covidence (www.covidence.org), an online platform of article screening and data extraction. The first stage of screening based on article titles and abstracts is going on. Two reviewers are conducting the screening independently and a third reviewer has resolved any disputes. We aim to finish the screening, data extraction and analysis by 30 April 2021 and report the result via web-based interactive platform (to be developed) by 30 June 2021.

\section{REFERENCES}

1. GBD Risk Factor Collaborators. Global, regional, and national comparative risk assessment of 84 behavioural, environmental and occupational, and metabolic risks or clusters of risks for 195 countries and territories, 19902017: a systematic analysis for the Global Burden of Disease Study 2017. Lancet. 2018;392(10159):19231994. doi:10.1038/sj.bdj.2015.751 
2. Bilano V, Gilmour S, Moffiet T, et al. Global trends and projections for tobacco use, 1990-2025: an analysis of smoking indicators from the WHO Comprehensive Information Systems for Tobacco Control. Lancet. 2015;385(9972):966976. doi:10.1016/s0140-6736(15)60264-1

3. Gartner CE, Barendregt JJ, Hall WD. Predicting the future prevalence of cigarette smoking in Australia: how low can we go and by when? Tob Control. 2009;18(3):183-189. doi:10.1136/tc.2008.027615

4. Hoffman SJ, Mammone J, Rogers Van Katwyk S, et al. Cigarette consumption estimates for 71 countries from 1970 to 2015: systematic collection of comparable data to facilitate quasi-experimental evaluations of national and global tobacco control interventions. BMJ. 2019;365:12231. doi:10.1136/bmj.12231

5. Chung-Hall J, Craig L, Gravely S, Sansone N, Fong GT. Impact of the WHO FCTC over the first decade: a global evidence review prepared for the Impact Assessment Expert Group. Tob Control. 2019;28(Suppl 2):s119-s128. doi:10.1136/tobaccocontrol-2018-054389

6. World Health Organization. MPOWER: Advancing the WHO Framework Convention on Tobacco Control (WHO FCTC). https://www.who.int/cancer/prevention/ tobacco_implementation/mpower/en/. Accessed May 2, 2019.

7. Gravely S, Giovino GA, Craig L, et al. Implementation of key demand-reduction measures of the WHO Framework Convention on Tobacco Control and change in smoking prevalence in 126 countries: an association study. Lancet Public Health. 2017;2(4):e166-e174. doi:10.1016/s2468-2667(17)30045-2

8. Munabi-Babigumira S, Fretheim A, Overland S. Interventions for Tobacco Control in Low- and MiddleIncome Countries: Evidence from Randomised and QuasiRandomized Studies. Oslo, Norway: Knowledge Centre for the Health Services at The Norwegian Institute of Public Health (NIPH); 2012. PMID:28510404.

9. Bala MM, Strzeszynski L, Topor-Madry R. Mass media interventions for smoking cessation in adults. Cochrane Database Syst Rev. 2017(11):CD004704. doi:10.1002/14651858.cd004704.pub4

10. Barnes J, Dong CY, McRobbie H, Walker N, Mehta M, Stead LF. Hypnotherapy for smoking cessation. Cochrane Database Syst Rev. 2010(10):CD001008. doi:10.1002/14651858.cd001008.pub2

11. Cahill K, Lancaster T. Workplace interventions for smoking cessation. Cochrane Database Syst Rev. 2014;(2):CD003440. doi:10.1002/14651858.CD003440.pub4

12. Sinclair HK, Bond CM, Stead LF. Community pharmacy personnel interventions for smoking cessation. Cochrane Database Syst Rev. 2004;(1):CD003698. doi:10.1002/14651858.CD003698.pub2

13. Ussher MH, Taylor AH, Faulkner GEJ. Exercise interventions for smoking cessation. Cochrane Database Syst Rev. 2014(8):(8):CD002295. doi:10.1002/14651858.cd002295.pub5

14. White AR, Rampes H, Liu JP, Stead LF, Campbell J. Acupuncture and related interventions for smoking cessation. Cochrane Database Syst Rev. 2011;(1):CD000009. doi:10.1002/14651858.CD000009.pub3

15. Whittaker R, McRobbie H, Bullen C, Rodgers A, Gu Y. Mobile phone-based interventions for smoking cessation. Cochrane Database Syst Rev. 2016;4(4):CD006611. doi:10.1002/14651858.CD006611.pub4

16. Cahill K, Stevens S, Perera R, Lancaster T. Pharmacological interventions for smoking cessation: an overview and network meta-analysis. Cochrane Database Syst Rev. 2013;(5):CD009329. doi:10.1002/14651858.cd009329.pub2

17. Lancaster T, Stead LF. Individual behavioural counselling for smoking cessation. Cochrane Database Syst Rev. 2017;3(3):CD001292. doi:10.1002/14651858.cd001292.pub2

18. Stead LF, Koilpillai P, Fanshawe TR, Lancaster T. Combined pharmacotherapy and behavioural interventions for smoking cessation. Cochrane Database Syst Rev. 2012;10:CD008286. doi:10.1002/14651858.cd008286.pub3

19. Coppo A, Galanti MR, Giordano L, Buscemi D, Bremberg S, Faggiano F. School policies for preventing smoking among young people. Cochrane Database Syst Rev. 2014;2014(10):CD009990. doi:10.1002/14651858.CD009990.pub2

20. Hefler M, Liberato SC, Thomas DP. Incentives for preventing smoking in children and adolescents. Cochrane Database Syst Rev. 2017;6(6):CD008645. doi:10.1002/14651858.cd008645.pub3

21. Wolfenden L, Goldman S, Stacey FG, et al. Strategies to improve the implementation of workplace-based policies or practices targeting tobacco, alcohol, diet, physical activity and obesity. Cochrane Database Syst Rev. 2018;11(11):CD012439. doi:10.1002/14651858.cd012439.pub2

22. Cahill K, Hartmann-Boyce J, Perera R. Incentives for smoking cessation. Cochrane Database Syst Rev. 2019;7(7):CD004307. doi:10.1002/14651858.CD004307.pub6

23. Hamilton FL, Greaves F, Majeed A, Millett G. Effectiveness of providing financial incentives to healthcare professionals for smoking cessation activities: systematic review. Tob Control. 2013;22(1):3-8. doi:10.1136/tobaccocontrol-2011-050048

24. van den Brand FA, Nagelhout GE, Reda AA, et al. Healthcare financing systems for increasing the use of tobacco dependence treatment. Cochrane Database Syst Rev. 2017;9(9):CD004305. doi:10.1002/14651858.CD004305.pub5

25. Snilstveit B, Vojtkova M, Bhavsar A, Stevenson J, Gaarder M. Evidence \& Gap Maps: A tool for promoting evidence informed policy and strategic research agendas. J Clin Epidemiol. 2016;79:120-129. doi:10.1016/j.jclinepi.2016.05.015

26. Munar W, Snilstveit B, Stevenson J, et al. Evidence gap map of performance measurement and management in primary care delivery systems in low- and middle-income countries - Study protocol. Gates Open Res. 2018;2:27. doi:10.12688/gatesopenres.12826.2

27. International Initiative for Impact Evaluation. Present your EGM online. http://www.3ieimpact.org/evidence- 
hub/evidence-gap-maps/open-access-egm. Accessed April 30, 2019.

28. Haddaway NR, Macura B, Whaley P, Pullin AS. ROSES RepOrting standards for Systematic Evidence Syntheses: pro forma, flow-diagram and descriptive summary of the plan and conduct of environmental systematic reviews and systematic maps. Environmental Evidence. 2018;7(1):7. doi:10.1186/s13750-018-0121-7

29. Specialist Unit for Review Evidence, Cardiff University. Critical appraisal checklists. http://www.cardiff.ac.uk/ specialist-unit-for-review-evidence/resources/criticalappraisal-checklists. Accessed June 16, 2020.

30. Schlam TR, Baker TB. Interventions for Tobacco Smoking. Annual Review of Clinical Psychology. 2013;9(1):675702. doi:10.1146/annurev-clinpsy-050212-185602

PROTOCOL REGISTRATION

The protocol is registered with PROSPERO (registration number: CRD42020135667). Also, it was pre-registered in Open Science Framework registry (https://osf.io/registries).

\section{CONFLICTS OF INTEREST}

The authors have completed and submitted the ICMJE Form for Disclosure of Potential Conflicts of Interest and none was reported.

FUNDING

There was no source of funding for this research.

PROVENANCE AND PEER REVIEW

Not commissioned; externally peer reviewed. 